\title{
Mutation patterns in gyrA and parC genes of ciprofloxacin resistant isolates of Neisseria gonorrhoeae from India
}

\author{
U Chaudhry, K Ray, M Bala, D Saluja
}

Sex Transm Infect 2002;78:440-444

See end of article for authors' affiliations

Correspondence to:

Dr Daman Saluja, 8/5,

Block \#4 1, Singh Sabha

Road, Delhi-1 10007 ,

India;

dsalujach@yahoo.com

Accepted for publication 22 April 2002

\begin{abstract}
Aim: To analyse mutations in the gyrA and parC genes leading to possible increase in ciprofloxacin resistance (high MIC values for ciprofloxacin) in clinical isolates of Neisseria gonorrhoeae in Delhi, India.

Method: MIC of ciprofloxacin for 63 clinical isolates of $\mathrm{N}$ gonorrhoeae were examined by the Etest method. Subsequently, gyrA and par $C$ genes of these isolates were amplified and sequenced for possible mutations.

Results: Out of the 63 clinical isolates tested, only five $(8 \%)$ isolates were found to be susceptible to ciprofloxacin (MIC $<0.06 \mu \mathrm{g} / \mathrm{ml})$. DNA sequence analysis of the gyrA and the parC genes of all these isolates $(n=63)$ revealed that all isolates which were not susceptible to ciprofloxacin $(n=58)$ had mutation(s) in gyrA and parC genes. 12 isolates (19\%) exhibited high resistance with an MIC for ciprofloxacin of $32 \mathrm{\mu g} / \mathrm{ml}$. Two out of these 12 isolates (UD62 and UD63), harboured triple mutations (Ser-91 to Phe, Asp-95 to Asn and Val-120 to Leu) in the gyrA gene. The third mutation of Val-120 to Leu, lies downstream of the quinolone resistance determining region (QRDR) of the gyrA and has not been described before in gonococcus. In addition, both these isolates had a Phe-100 to Tyr substitution in the parC, a hitherto unknown mutation.

Conclusions: Emergence of ciprofloxacin resistance with high levels of MIC values (up to $32 \mu \mathrm{g} / \mathrm{ml}$ ) in India is alarming. Double and triple mutations in gyrA alone or together in gyrA and parC could be responsible for such a high resistance.
\end{abstract}

N eisseria gonorrhoeae, a common causative agent of sexually transmitted diseases (STD), is still prevalent in high numbers in developing countries. ${ }^{12}$ Owing to the emergence of penicillin and tetracycline resistant strains of $N$ gonorrhoeae, CDC and WHO recommended third generation cephalosporins and fluoroquinolones for the treatment of gonococcal infections worldwide, including India. ${ }^{3}{ }^{4}$ However, the decreased susceptibilities of $N$ gonorrhoeae to fluoroquinolones has recently been reported from a large number of countries. $^{5-12}$ Ciprofloxacin, a fluoroquinolone, inhibits DNA replication by targeting DNA gyrase and topoisomerase IV. ${ }^{13}{ }^{14}$ In laboratory mutants of $N$ gonorrhoeae, mutations in the gyrA and gyrB genes of DNA gyrase and in the parC gene of topoisomerase IV, associated with decreased susceptibilities to quinolones, have been identified.${ }^{15-18}$ An amino acid change in the GyrB tends to confer a low level resistance to nalidixic acid. ${ }^{16}$ Alterations in the ParC have been identified only in strains having GyrA mutations and had a complementary role in further decreasing susceptibility of $N$ gonorrhoeae to fluoroquinolones. ${ }^{11} 151718$

The present study was performed to examine the susceptibility to ciprofloxacin of $N$ gonorrhoeae isolated from clinical samples $(n=63)$ collected from patients visiting Safdarjung Hospital, Delhi, India, from January 2000 to March 2001. The studies were further extended to understand the sharp increase in ciprofloxacin resistance, observed in Delhi, and to find out a correlation between mutation patterns in gyrA and the parC genes and the level of ciprofloxacin resistance.

\section{MATERIALS AND METHODS}

\section{Gonococcal isolates}

A total of 78 male patients with signs and symptoms of acute gonococcal urethritis attending the STD Teaching and
Research Centre of Safdarjung Hospital in New Delhi, India, from January 2000 to March 2001 were investigated for the presence of Gram negative intracellular diplococci in their urethral discharge. A total of 63 samples were smear positive. Specimens from these smear positive patients were inoculated directly onto Thayer-Martin selective agar (Hi Media, India), and incubated for $24-48$ hours at $35^{\circ} \mathrm{C}$ in a humid atmosphere containing $5-10 \% \mathrm{CO}_{2}$ (candle extinction jar). ${ }^{19}$ The culture positive isolates were further confirmed as $N$ gonorrhoeae by colony morphology, Gram staining, oxidase test, and carbohydrate degradation test. ${ }^{19}$ Post-treatment isolates or repeat isolates from the same patients were excluded in this study.

\section{Susceptibility testing}

The MIC of ciprofloxacin was determined by the Etest method in all the culture positive isolates ( 63 cultures) using the steps suggested by the manufacturer (AB Biodisks, Solna, Sweden). An inoculum of $10^{7} \mathrm{cfu} / \mathrm{ml}$ was allowed to grow on chocolate agar medium for 24 hours in a candle extinction jar. ${ }^{19}$ Based on MIC values, the strains were labelled as susceptible (MIC $<0.06 \mu \mathrm{g} / \mathrm{ml}$ ), intermediate resistant (MIC $0.06 \mu \mathrm{g} / \mathrm{ml}$ to 0.75 $\mu \mathrm{g} / \mathrm{ml}$ ), and resistant $(\mathrm{MIC} \geqslant \mathrm{l} \mu \mathrm{g} / \mathrm{ml}) .{ }^{20}$ WHO A-E, H, and $\mathrm{J}$ strains were used as controls. ${ }^{21}$

\section{Molecular study}

The gyrA and parC genes of each of the gonococcal isolate were amplified using oligonucleotide primers as reported by Tanaka et al. ${ }^{11}$ These primers amplified gyrA gene from nucleotides 160-438 (278 bp product), corresponding to the amino acids 54-146. The 255 bp amplified product of parC gene represented the nucleotides 166-420, which corresponds to amino acids 56-140. ${ }^{15}$ Chromosomal DNA was extracted by standard methods $\mathrm{s}^{22}$ and the PCR amplification was performed in $50 \mu \mathrm{l}$ of a reaction mixture which contained $5 \mu \mathrm{l}$ of 10x Taq 


\begin{tabular}{|c|c|c|c|c|c|c|}
\hline \multirow[b]{2}{*}{ Name of isolates } & \multirow[b]{2}{*}{ No of isolates } & \multirow[b]{2}{*}{$\%$ isolates } & \multirow{2}{*}{\multicolumn{2}{|c|}{$\begin{array}{l}\mathrm{MIC}^{*}(\mathrm{\mu g} / \mathrm{ml}) \text { of } \\
\text { ciprofloxacin }\end{array}$}} & \multicolumn{2}{|c|}{ No of mutations in } \\
\hline & & & & & gyrA & parC \\
\hline \multicolumn{7}{|l|}{ Clinical isolates } \\
\hline UD1-UD5 & 5 & $8 \%$ & $<0.06$ & (S) & No mutation & No mutation \\
\hline UD6 & 1 & $1 \%$ & 0.1 & (IR) & One & No mutation \\
\hline UD7-UD9 & 3 & $5 \%$ & 0.25 & (IR) & Two & One (silent) \\
\hline UD10-UD13 & 4 & $6 \%$ & 0.38 & (IR) & Two & One (silent) \\
\hline UD14-UD21 & 8 & $13 \%$ & 0.50 & (IR) & Two & One (silent) \\
\hline UD22-UD28 & 7 & $11 \%$ & 0.75 & (IR) & Two & Two (silent) \\
\hline UD29-UD32 & 4 & $6 \%$ & 1.0 & (R) & Two & One \\
\hline UD33-UD40 & 8 & $13 \%$ & 1.5 & (R) & Two & One \\
\hline UD4 1-UD44 & 4 & $6 \%$ & 2.0 & (R) & Two & One \\
\hline UD45, UD46 & 2 & $3 \%$ & 3.0 & (R) & Two & One \\
\hline UD47-UD50 & 4 & $6 \%$ & 4.0 & (R) & Two & One \\
\hline UD51 & 1 & $1 \%$ & 12.0 & (R) & Two & Two (one silent) \\
\hline UD52-UD61 & 10 & $16 \%$ & 32.0 & (R) & Two & Two (one silent) \\
\hline UD62, UD63 & 2 & $3 \%$ & 32.0 & (R) & Three & Three (two silent) \\
\hline \multicolumn{7}{|c|}{ WHO reference strains: } \\
\hline WHO strain A & 1 & - & 0.016 & (S) & No mutation & No mutation \\
\hline WHO strain $\mathrm{H}$ & 1 & - & 2.0 & (R) & Two & One \\
\hline WHO strain J & 1 & - & 8.0 & (R) & Two & One \\
\hline
\end{tabular}

*Based on MICs (minimum inhibitory concentrations), isolates were labelled as susceptible (S), intermediate resistant (IR), and resistant (R). ${ }^{20}$

polymerase buffer ( $500 \mathrm{mM} \mathrm{KCl,} 100 \mathrm{mM}$ TRIS- $\mathrm{HCl}(\mathrm{pH} 8.3)$, $15 \mathrm{mM} \mathrm{MgCl}_{2}, 0.1 \%$ gelatin), $25 \mathrm{pmol}$ of each of the two primers (reverse and forward), $200 \mu \mathrm{M}$ of each of the four deoxynucleoside triphosphates (New England Biolabs, Inc, USA), 1.5 U of Taq DNA polymerase (BangaloreGenei India Pvt Ltd, India), and $100 \mathrm{ng}$ of template DNA. Thirty five cycles were performed for each reaction. Each cycle consisted of 30 seconds at $93^{\circ} \mathrm{C}, 1$ minute at $52^{\circ} \mathrm{C}$, and 1 minute at $72^{\circ} \mathrm{C}$. The amplified PCR product was separated by electrophoresis on agarose gel and eluted from agarose by using a DNA isolation kit from Biological Industries, Israel, as in the manufacturer's instructions. The purified DNA was air dried and suspended in $12-15 \mu \mathrm{l}$ of sterile water. Gel purified PCR amplified product ( $100 \mathrm{ng}$ ) of gyrA gene was digested with Hinfl (l unit) in a final volume of $10 \mu \mathrm{l}$ for 2 hours at $37^{\circ} \mathrm{C}$. The digests were subjected to electrophoresis on 1.5\% agarose gel and visualised by ethidium bromide staining. Purified PCR amplified product of gyrA (corresponding to 160-438 nucleotides) and parC (corresponding to 166-420 nucleotides) genes of all the isolates as well as WHO reference strains $\mathrm{A}, \mathrm{H}$, and $\mathrm{J}$, were subjected to DNA sequencing using PCR primers (forward primer) with a Taq-Dye Terminator cycle sequencing kit and a Model 377A autosequencer (Applied Biosystems) as in the manufacturer's instructions. The region sequenced includes the quinolone resistance determining region (QRDR) of the GyrA protein (amino acids 55 to 110 ) and the ParC protein (amino acids 66-119). DNA sequences were analysed using the BLAST program.

\section{RESULTS}

\section{Susceptibilities of clinical isolates to ciprofloxacin}

Table 1 shows the MIC values of ciprofloxacin for the 63 gonococcal isolates. The susceptibility pattern of $N$ gonorrhoeae isolates was based on criteria proposed by Knapp et al. ${ }^{20}$ Only five isolates $(8 \%)$ were found to be susceptible to ciprofloxacin with an MIC $<0.06 \mu \mathrm{g} / \mathrm{ml}$. Out of the 58 quinolone resistant $N$ gonorrhoeae (QRNG) isolates, 23 (36\%) isolates were exhibiting intermediate resistance towards ciprofloxacin (MIC values ranging from $0.06-0.75 \mu \mathrm{g} / \mathrm{ml}$ ), while 35 isolates (56\%) were resistant to ciprofloxacin with a wide range of MIC (1-32 $\mu \mathrm{g} / \mathrm{ml}$ ). Among these 35 resistant isolates, 12 isolates (35\%) exhibited high levels of resistance with an MIC for ciprofloxacin of $32 \mu \mathrm{g} / \mathrm{ml}$.

\section{Hinf1 digestion to screen mutations in gyrA gene}

Quick screening of all the isolates, for the mutation at the Ser9lcodon in gyrA gene, was done by Hinfl restriction endonuclease digestion. PCR amplified gyrA gene product (278 bp) of all the isolates, which were susceptible to ciprofloxacin, when digested with the Hinfl restriction endonuclease enzyme gave two bands of $165 \mathrm{bp}$ and $113 \mathrm{bp}$ on the agarose gel (fig l). However, PCR products of gyrA gene, amplified from all other isolates, were not digested with Hinfl. The amplified products of the gyrA gene of WHO reference strains $\mathrm{H}$ and J (resistant to ciprofloxacin) were also resistant to digestion with the Hinfl enzyme while that of WHO strain A (sensitive to ciprofloxacin) gave two bands of $165 \mathrm{bp}$ and $113 \mathrm{bp}$ (fig 1). Thus, all the gonococcal isolates, resistant to ciprofloxacin $(n=58)$, were found to be mutated at Ser-91 position within QRDR of GyrA protein.

\section{Novel mutations in gyrA and parC genes in isolates showing high resistance to ciprofloxacin}

DNA sequencing of the PCR amplified region of the gyrA and the parC genes from the ciprofloxacin susceptible isolates (UD1-UD5), revealed no alteration in either of the genes (table 2). However, multiple mutations were observed in the PCR amplified region of gyrA and the parC genes from the QRNG isolates $(\mathrm{n}=58)$. All these isolates had mutation in the codon 91 of the gyrA leading to Ser-91 to Phe substitution. Single mutation, at position 91 of GyrA, was observed in only one isolate, exhibiting intermediate resistance towards ciprofloxacin with an MIC of $0.1 \mu \mathrm{g} / \mathrm{ml}$ (table 2). All other QRNG isolates $(\mathrm{n}=57)$ had atleast two mutations in the gyrA gene along with parC mutation(s). The most frequent mutations (in $81 \%$ of QRNG isolates) are the Ser-91 to Phe and Asp-95 to Asn substitutions in the GyrA protein. Among the 12 isolates with MIC for ciprofloxacin of $32 \mu \mathrm{g} / \mathrm{ml}, 10$ isolates (UD52 to UD61) had an Asp-95 to Gly substitution along with the Ser-91 to Phe in the gyrA gene. The other two isolates, UD62 and UD63, had three point mutations in the gyrA gene resulting in three amino acid substitutions (Ser-91 to Phe, Asp-95 to Asn, and Val-120 to Leu) in the GyrA (table 2). Interestingly this third mutation (Val-120 to Leu) lies outside the so far recognised QRDR (amino acids 55 to 110 of $N$ gonorrhoeae GyrA protein). ${ }^{15}$ WHO strain A, which is sensitive to ciprofloxacin (MIC of $0.016 \mu \mathrm{g} / \mathrm{ml}$ ), had no mutation in the gyrA gene while WHO strain $\mathrm{H}$ (MIC of $2.0 \mu \mathrm{g} / \mathrm{ml}$ for ciprofloxacin) and WHO 


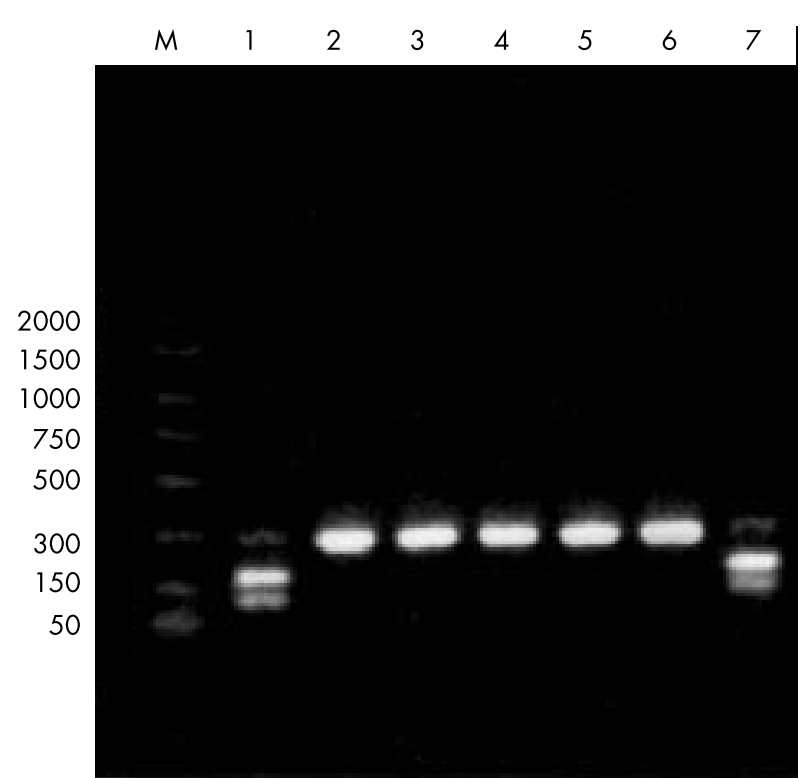

Figure 1 Hinfl restriction fragment length polymorphism. Region spanning QRDR of gyrA gene of Neisseria gonorrhoeae was amplified and subjected to Hinfl restriction enzyme digestion. Ciprofloxacin susceptible isolates (lane 1; susceptible clinical isolate, lane 7; WHO reference strain A) with no mutation produced two fragments of $165 \mathrm{bp}$ and $113 \mathrm{bp}$ on Hinfl digestion. The ciprofloxacin resistant isolates (lanes 2-4; QRNG clinical isolates, lane 5; WHO reference strain $\mathrm{H}$, lane 6; WHO reference strain J) remain undigested as Hinfl cleavage site is lost because of the $C$ to T transition at nucleotide position 272 (Ser-91 to Phe). Lane M; 100 bp molecular weight standard.

strain J (MIC of $8 \mu \mathrm{g} / \mathrm{ml}$ for ciprofloxacin), had two mutations leading to Ser-91 to Phe and Asp-95 to Gly substitutions.

Sequence analysis of the parC gene demonstrated a variety of mutations at codons 91, 100, 104, 109, and 131 (table 2). The isolates (UD7-UD28), exhibiting intermediate resistance to ciprofloxacin (MIC from 0.06-0.75 $\mathrm{g} / \mathrm{ml}$ ), had only silent mutations at the parC locus. The most common substitution of Glu-91 to Gly in the parC gene was found in 33 QRNG isolates (57\%). The two isolates (UD62, UD63), having a novel mutation in the gyrA gene, also possessed a hitherto undescribed mutation at the parC, resulting in the substitution of Phe-100 with Tyr. Along with this mutation, two silent mutations at codon 104 (Tyr) and 109 (Gly) were also observed in the parC gene of these two isolates. The silent mutation at the codon 109 has also not been described before. WHO strain A (MIC of $0.016 \mu \mathrm{g} / \mathrm{ml}$ ) had no mutation in the parC gene while WHO Strain H (MIC of $2.0 \mu \mathrm{g} / \mathrm{ml}$ for ciprofloxacin) and WHO strain J (MIC of $8 \mu \mathrm{g} / \mathrm{ml}$ for ciprofloxacin) possessed a single mutation at the codon 88 (Ser-88 to Pro) and 86 (Asp-86 to Asn) respectively.

\section{DISCUSSION}

During the past few years, an overall increase in resistance to various antimicrobial agents have been observed in $N$ gonorrhoeae. ${ }^{6} 102324$ Despite a high prevalence of gonorrhoea in India, a systematic study regarding resistance to various antimicrobial agents is limited. ${ }^{702325}$ In the present study, an increase in the number of QRNG isolates (92\%) as well as the level of resistance to ciprofloxacin (MIC up to $32 \mu \mathrm{g} / \mathrm{ml}$ ) was observed in $N$ gonorrhoeae isolated from patients visiting STD Centre, Safdarjung Hospital, India. A significant proportion of high level QRNG (63\%) has also been reported from Philippines. ${ }^{2}$ Almost 90\% of isolates in China and Hong Kong were QRNG and about half of these isolates possessed high level of resistance. ${ }^{2}$ In Japan, although the overall percentage

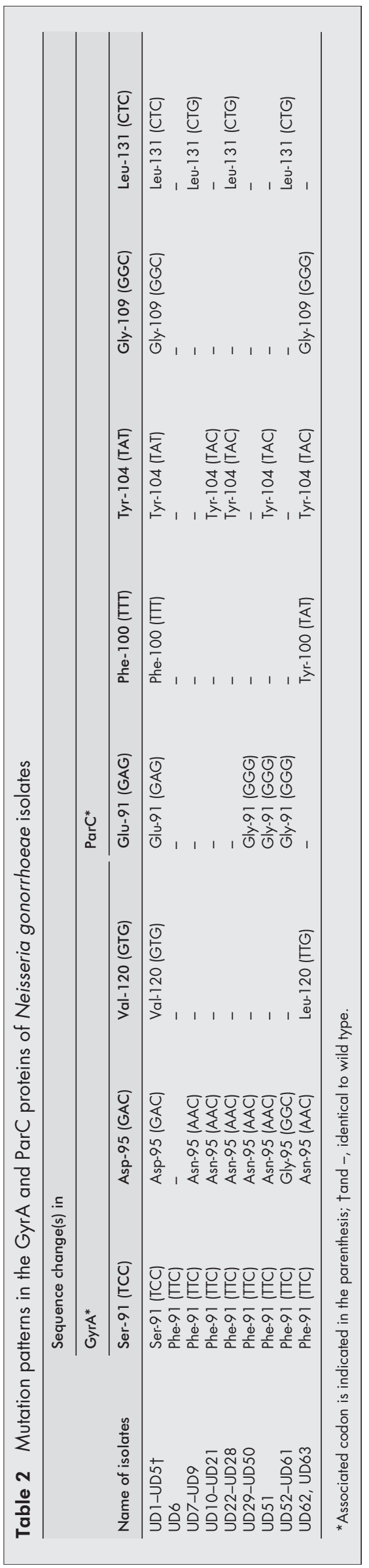


of resistant strains has not changed appreciably in past 2 years, there was a gradual increase in level of resistance (MIC $>1.0 \mu \mathrm{g} / \mathrm{ml}) .{ }^{11}$ Decreased susceptibility to ciprofloxacin and ofloxacin has also been reported from Bangladesh (11.7\% were resistant and $26.6 \%$ had reduced susceptibility). ${ }^{9}$ This widespread emergence of ciprofloxacin resistance in South East Asia is rather alarming.

Mutations in the gyrA and the parC genes leading to amino acid changes in GyrA (especially at Ser-9l and Asp-95) and ParC proteins are significantly associated with ciprofloxacin resistance. ${ }^{511} 151718$ 26-28 Mutation at codon 91 of the gyrA gene results in the loss of natural Hinfl site. Direct Hinfl digestion of PCR amplified gyrA gene has been used to screen gyrA mutations in $N$ gonorrhoeae. ${ }^{27}{ }^{29}$ Another restriction endonuclease site $(A l w 1)$ spans the novel mutation (Val-120 to Leu), identified by us. Double digestion with Hinfl and Alw1 would result in release of a fragment from the wild type but not mutated gyrA gene and may be used to check for mutations at these two loci.

Since QRNG isolates (intermediate and resistant) exhibit a wide range of MIC for ciprofloxacin $(0.1-32 \mu \mathrm{g} / \mathrm{ml})$, mutation pattern in gyrA and parC genes was checked in all the isolates. Double and triple mutations in the QRDR region of gyrA and the parC genes appear to be the major contributors to the high level of ciprofloxacin resistance. It is significant to note that the most common gyrA mutations (in $75 \%$ of the isolates) found by us were Ser-91 to Phe and Asp-95 to Asn, while only $16 \%$ isolates showed change of Asp-95 to Gly along with Ser-91 to Phe. None of the isolates sequenced in this study had tyrosine at position 91 in the gyrA gene. ${ }^{11}{ }^{12}{ }^{28}$ We also did not observe any isolate carrying mutation at Ala-67, Ala-75, and Ala-84 in the gyrA, as reported in some of the isolates from Japan. ${ }^{11}$ Isolates (UD7-UD28), with low MIC for ciprofloxacin $(0.25-0.75 \mu \mathrm{g} / \mathrm{ml})$, had silent mutations at the parC locus, while all other isolates (MIC of $1-32 \mu \mathrm{g} / \mathrm{ml}$ ), except UD62 and UD63, had Glu-91 to Gly substitution. This supports the earlier observations that mutations in parC have a complementary role in decreasing susceptibility to ciprofloxacin. ${ }^{11} 1517182627$ Alterations at the Gly-85, Asp-86, Ser-87, Ser-88, or Arg 116 residues, in parC, as reported in the earlier studies, ${ }^{11} 1727$ have not been found in any of the clinical isolates in the present study. Instead, two isolates (UD62 and UD63) with high MIC for ciprofloxacin had a Phe-100 to Tyr substitution along with the triple mutations in the QRDR of the GyrA protein (Ser-9l to Phe, Asp-95 to Asn, and Val-120 to Leu). The third mutation observed in these two isolates has not been reported so far. Though close to QRDR in the GyrA subunit, this novel mutation is present downstream of the identified QRDR. Additional mutations in the gyrA gene, about 20-30 amino acids away from Ser-83 in Escherichia coli and Thr-83 in Pseudomonas aeruginosa, leading to quinolone resistance, have also been reported in E coli (Gln-106 to Arg, Gln-106 to His) and P aeruginosa (Asn-116 to Tyr) respectively. ${ }^{30}{ }^{31}$ Acknowledging that transformation studies need to be performed for confirmation, these results suggest that triple mutations in gyrA along with the Phe-100 to Tyr substitution in the parC may have some bearing on the action of ciprofloxacin in $N$ gonorrhoeae.

The results of our study clearly indicate that resistance to ciprofloxacin, which was introduced as first line therapy only in 1990, has increased rapidly with an alarming high level of MIC values and new mutational patterns in $N$ gonorrhoeae. Unless continued monitoring of antimicrobial sensitivity patterns of gonococcal strains circulating in a community is carried out, treatment failures may occur and lead to prolonged infection and spread of multiple drug resistant strains of $N$ gonorhoeae.

\section{ACKNOWLEDGEMENTS}

We are grateful to the director, Centre for Biochemical Technology, for extending their facilities for DNA sequencing to us. We thank Ms

\section{Key messages}

- Emergence of ciprofloxacin resistant Neisseria gonorrhoeae

- High percentage of isolates have high level of MIC for ciprofloxacin

- Characterisation of novel mutations in the QRDR region of gyrA and parC genes of Neisseria gonorrhoeae

- Rapid screening of ciprofloxacin resistant isolates by Hinfl digestion of the gyrA amplified product.

Leelamma Peter for excellent technical assistance. One of us (UC) is grateful to CSIR for the award of senior research fellowship.

\section{CONTRIBUTORS}

KR (head, STD laboratory) and MB collected the clinical samples and conducted the drug sensitivity for Neisseria gonorrhoeae; UC carried out PCR and analysis of mutations in the QRDR region of gyrA and parC genes; DS instigated the project, designed the study, and analysed the data; DS and UC wrote the manuscript.

\section{Authors' affiliations}

U Chaudhry, D Saluja, Dr BR Ambedkar Center for Biomedical Research, University of Delhi, Delhi-1 10007, India

K Ray, M Bala, Regional STD Laboratory, Safdarjung Hospital, New Delhi, India

\section{REFERENCES}

1 WHO Western Pacific Region Gonococcal Surveillance Programme. Surveillance of antibiotic susceptibility of Neisseria gonorrhoeae in the WHO western pacific region 1992-94. Genitourin Med 1997;73:355-61.

2 WHO Western Pacific Region Gonococcal Surveillance Programme. Surveillance of antibiotic resistance in Neisseria gonorrhoeae in the WHO western pacific region, 1998. Comm Dis Intell 2000;24:1-4

3 Center for Disease Control and Prevention. Sexually transmitted disease treatment guidelines. MMWR Morb Mortal Wkly Rep 1989;38:S8

4 STD Treatment Strategies. WHO consultation on development of sexually transmitted disease treatment strategies. (WHO/VDT/89-477) 1989.

5 Deguchi T, Yasuda M, Asano $M$, et al. DNA gyrase mutations in quinolone resistant clinical isolates of Neisseria gonorrhoeae. Antimicrob Agents Chemother 1995;39:561-3.

6 Fox KK, Knapp JS, Holmes KK, et al. Antimicrobial resistance in Neisseria gonorrhoeae in the United States, 1988-1994: the emergence of decreased susceptibility to fluoroquinolones. J Infect Dis 1997; 175: 1396-403

7 Rattan A, Kumari S, Khanna N, et al. Emergence of fluoroquinolone resistant Neisseria gonorrhoeae from New Delhi, India. Sex Transm Infect 1998;74:229

8 Lee K, Chong Y, Erdenechemeg L, et al. Incidence, epidemiology and evolution of reduced susceptibility to ciprofloxacin in Neisseria gonorrhoeae in Korea. Clin Microbiol Infect 1998:4:627-33.

9 Bhuiyan BU, Rahman M, Amin Miah, et al. Antimicrobial susceptibilities and plasmid contents of Neisseria gonorrhoeae isolates from commercia sex workers in Dhaka, Bangladesh: emergence of high-level resistance to ciprofloxacin. J Clin Microbiol 1999;37:1130-6

10 Ray K, Bala M, Kumar J, et. al. Trend of antimicrobial resistance in Neisseria gonorrhoeae at New Delhi, India. Int J STD AIDS 2000;11:115-18.

11 Tanaka M, Nakayama H, Haraoka, et al. Antimicrobial resistance of Neisseria gonorrhoeae and high prevalence of ciprofloxacin-resistant isolates in Japan, 1993 to 1998. J Clin Microbiol 2000;38:521-5.

12 Mavroidi A, Tzouvelekis LS, Tassios PT, et al. Characterization of Neisseria gonorrhoeae strains with decreased susceptibility to fluoroquinolones isolated in Greece from 1996-1999. J Clin Microbiol 2000;38:3489-91

13 Drlica K, Zhao X. DNA gyrase, topoisomerase IV, and the 4-quinolones. Microbiol Mol Biol Rev 1997:61:377-92.

14 Drlica K. Refining the fluoroquinolones. ASM News 1999;65:410-15.

15 Belland RJ, Morrison SG, Ison C, et al. Neisseria gonorrhoeae acquires mutations in analogous regions of gyrA and parC in fluoroquinolone-resistant isolates. Mol Microbiol 1994;14:371-80.

16 Stein DC, Danaher RJ, Cook TM. Characterization of a gyrB mutation responsible for low-level nalidixic acid resistance in Neisseria gonorrhoeae. Antimicrob Agents Chemother 1991;35:622-6.

17 Trees DL, Sandul AL, Whittington WL, et al. Identification of novel mutation patterns in the parC gene of ciprofloxacin-resistant isolates of Neisseria gonorrhoeae. Antimicrob Agents Chemother 1998;42:2103-5. 
18 Tanaka M, Nakayama H, Haraoka M et al. Susceptibilities of Neisseria gonorrhoeae isolates containing amino acid substitutions in GyrA, with or without substitutions in ParC, to newer fluoroquinolones and other antibiotics. Antimicrob Agents Chemother 2000;44:192-5.

19 Van Dyck E, Piot P, Meheus A. Bench level laboratory manual for STDs. WHO/VDT. 1989:443

20 Knapp JS, Hale JA, Neal SW, et al. Proposed criteria for interpretation of susceptibilities of strains of Neisseria gonorrhoeae to ciprofloxacin ofloxacin, enoxacin, lomefloxacin and norfloxacin. Antimicrob Agents Chemother 1995;39:2442-5.

21 World Health Organization. Reference Manual. Laboratory diagnosis of gonorrhoea. WHO regional publication, South-East Asia Series No 33. Geneva: WHO, 1999:33, 37

22 Ausubel FM, Brent R, Kingston RE, et al. Current protocols in molecular biology. New York: John Wiley, 1997;2:2.

23 Bhalla P, Sethi K, Reddy BSN, et al. Antimicrobial susceptibility and plasmid profile of Neisseria gonorrhoeae in India, New Delhi. Sex Transm Infect 1998;74:210-2

24 Lee K, Shin JW, Lim JB, et al. Emerging antimicrobial resistance, plasmid profile and pulse field gel electrophoresis pattern of the endonuclease-digested genomic DNA of Neisseria gonorrhoeae. Yonsei Med J 2000;41:381-6.
25 Pai A, Kulkarni MG, Koppikar GV. Antibiotics sensitivity pattern of Neisseria gonorrhoeae with special emphasis on norfloxacin and ciprofloxacin. J Assoc Physicians India 1999;47:795-7.

26 Deguchi T, Yasuda M, Nakano M, et al. Quinolone-resistant Neisseria gonorrhoeae: correlation of alterations in the GyrA subunit of DNA gyrase and the ParC subunit of topoisomerase IV with antimicrobial susceptibility profiles. Antimicrob Agents Chemother 1996;40:1020-3.

27 Trees DL, Sandul AL, Neal SW, et al. Molecular epidemiology of Neisseria gonorrhoeae exhibiting decreased susceptibility and resistance to ciprofloxacin in Hawaii, 1991-1999. Sex Transm Dis 2000;28:309-14

28 Ison CA, Woodford PJ, Madders H, et al. Drift in susceptibility of Neisseria gonorrhoeae to ciprofloxacin and emergence of therapeutic failure. Antimicrob Agents Chemother 1998;42:2919-22.

29 Deguchi T, Yasuda M, Nakano M, et al. Rapid detection of point mutations of the Neisseria gonorrhoeae gyrA gene associated with decreased susceptibilities to quinolones. J Clin Microbiol 1996;34:2255-8

30 Hallett P, Maxwell A. Novel quinolone resistance mutations of the Escherichia coli DNA gyrase A protein: enzymatic analysis of the mutant proteins. Antimicrob Agents Chemother 1991;35:335-40.

31 Lee $\mathrm{S}$, Lee $Y$. Ofloxacin resistance mechanism in PA150 and PA300-clinical isolates of Pseudomonas aeruginosa in Korea. Arch Pharm Res 1998;21:671-6.

\section{Multiple sclerosis may be sexually acquired}

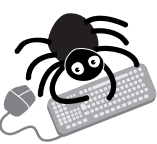

Please visit the Sexually Transmitted Infections website [www sextransinf.com] for link to this full article.
S research may change direction if researchers take up the challenge of testing the hypothesis that a recent review proposes-namely, that MS is a sexually transmitted infection (STI). The author Iemphasises that this may be just one way in which the disease occurs but maintains that there is sufficient evidence to suggest that it is spread sexually, manifesting itself in those who are genetically susceptible.

MS is generally assumed to have a genetic or immune basis, but it shares parallels with STIs-not least tropical spastic paraplegia-an STI whose similarity to primary progressive MS is striking.

Historically, clusters or epidemics of MS recorded after an influx of troops in communities with a previously low incidence of the disease-for example, Allied troops sent to Iceland, Shetland, and the Faroes in World War II-are consistent with the spread of an STI. MS is initially more prevalent in females, and is passed on more efficiently from men to women. Migration at a young age from low risk to high risk areas for MS rapidly increases risk status. Prevalence mirrors social and cultural norms: MS is most prevalent in permissive societies and least so in Moslem Arab countries, in ethnic populations living apart from white populations - Aborigines, Maoris - and in closed white communities with strict moral attitudes. It also mirrors changing social norms: the Danish MS Registry, with records from 1949, reported a significant increase in MS in women when oral contraceptives became available and use of barrier methods offering more protection against STIs declined.

A Journal of Neurology, Neurosurgery, and Psychiatry 2002:73:439-443. 